\title{
INTERPOLATION SPACES AND UNITARY REPRESENTATIONS
}

\author{
ROE GOODMAN ${ }^{1}$
}

\begin{abstract}
Let $\boldsymbol{G}$ be a Lie group, $\pi$ a unitary representation of $\boldsymbol{G}$ on a Hilbert space $\mathcal{H}(\pi)$, and $\mathcal{H}^{k}(\pi)$ the subspace of $C^{k}$ vectors for $\pi$. By quadratic interpolation there is a continuous scale $\mathcal{F C}^{\mathcal{J}}(\pi), s>0$, of $G$-invariant Hilbert spaces. When $G=H \cdot K$ is a semidirect product of closed subgroups, then it is proved that $\mathcal{H C}^{\mathcal{S}}(\pi)=\mathcal{H C}^{\mathcal{S}}\left(\left.\pi\right|_{H}\right) \cap \mathcal{H C}^{\mathcal{C}}\left(\left.\pi\right|_{K}\right)$ for $s>0$. For solvable $G$ this gives a characterisation of $\mathcal{H C}^{3}(\pi)$ in terms of smoothness along one-parameter subgroups, and an elliptic regularity result.
\end{abstract}

1. Introduction. Let $G$ be a Lie group, $g$ its Lie algebra, and $U(\mathfrak{g})$ the complexified universal enveloping algebra of $g$. If $\pi$ is a strongly continuous representation of $G$ on a Banach space $\mathcal{H}(\pi)$, one has for each positive integer $k$ the subspace $\mathcal{H}^{k}=\mathcal{H}^{k}(\pi)$ of $C^{k}$ vectors for $\pi$, and the space $\mathcal{H}^{\infty}(\pi)=\cap \mathcal{H}^{k}$ of $C^{\infty}$ vectors. These spaces are $G$-invariant. Furthermore, $\mathcal{H}^{k}$ can be normed as a Banach space so that $\left.g \mapsto \pi(g)\right|_{\mathcal{C}^{k}}$ is strongly continuous (cf. [3]).

It is natural to consider interpolation spaces between $\mathcal{F C}^{k}$ and $\mathcal{F C}^{k+1}$, constructed either by the "complex" or "real" method. For general Banach space representations, this gives a profusion of different spaces (cf. [2], [10] for the much-studied case $G=\mathbf{R}^{n}$ ). For unitary representations one may use the "quadratic interpolation functor" [9] to obtain a continuous interpolating scale of Hilbert spaces $\mathcal{H C}^{3}$, $s>0$ (cf. [4]), which are $G$-invariant. (For noncommutative $G$, however, the $G$ action on $\mathscr{H}^{s}$ is nonunitary for $s>0$, when the adjoint representation is nonunitary.) The main result of this note is the following

REDUCTION THEOREM. If $G=H \cdot K$ is a semidirect product of closed subgroups $H$ and $K$, then for any unitary representation $\pi$ and $s>0$,

$$
\mathcal{H}^{s}(\pi)=\mathcal{H}^{s}\left(\left.\pi\right|_{H}\right) \cap \mathcal{H}^{s}\left(\left.\pi\right|_{K}\right) \text {. }
$$

We shall prove this theorem in $\$ 5$, using basic properties of diffusion semigroups generated by Laplace operators, which are recalled in $\$ 3$, and a "noncommutative interpolation" theorem of P. Grisvard [7], which is stated in the form we need it in §4. This approach seems quite natural, in view of the fact that for $s>0, \mathcal{H}^{3}(\pi)$ is the domain of the operator $A^{s / 2}$, where $A$ is the closure of $\pi_{\infty}(-\Delta), \Delta$ a Laplace operator on $G$ (cf. $\$ 5$ for notation).

2. Applications. We turn now to some consequences of the Reduction Theorem, when $G$ is solvable. First we describe $\mathscr{H}^{s}(\pi), 0<s<1$, in the case $G=\mathbf{R}$. Here the result is known (cf. [10, Proposition 4, p. 139]).

Received by the editors January 23, 1980 and, in revised form, January 19, 1981.

AMS (MOS) subject classifications (1970). Primary 22E45, 22E30; Secondary 46E35.

${ }^{1}$ Research partially supported by NSF Grant MCS 76-07097.

(C) 1981 American Mathematical Society 0002-9939/81/0000-0435/\$02.50 
Proposition 2.1. If $\pi$ is a unitary representation of $\mathbf{R}$ and $0<s<1$, then $u \in \mathcal{H}^{s}(\pi)$ if and only if

$$
\int_{-\infty}^{\infty}\|\pi(x) u-u\|^{2}|x|^{-2 s-1} d x<\infty
$$

Proof. By Bochner's theorem, there is a unique positive finite measure $\mu$ on $\mathbf{R}$ such that

$$
(\pi(x) u, u)=\int_{-\infty}^{\infty} e^{i x \xi} d \mu(\xi)
$$

Thus the left side of (2.1) is given by

$$
\int_{-\infty}^{\infty}\left\{\int_{-\infty}^{\infty}\left|e^{i x \xi}-1\right|^{2}|x|^{-2 s-1} d x\right\} d \mu(\xi)
$$

Making the change of variable $x \rightarrow \xi^{-1} x$ in the inner integral, one obtains the integral

$$
C_{s} \int_{-\infty}^{\infty}|\xi|^{2 s} d \mu(\xi)
$$

with $C_{s} \neq 0$. But (2.2) is finite if and only if $u \in \mathcal{H}^{s}(\pi)$. Q.E.D.

Suppose now that $G$ is solvable. Let $\left\{X_{j} \mid 1<j<n\right\}$ be a Jordan-Hölder basis for g, i.e. $\left[X_{j}, \mathfrak{g}_{j+1}\right] \subseteq \mathfrak{g}_{j+1}$, where $\mathfrak{g}_{j}=\operatorname{span}\left\{X_{i} \mid i>j\right\}$. From the Reduction Theorem and Proposition 2.1 we obtain

THEOREM 2.2. Let $\pi$ be a unitary representation of $G, \pi_{j}$ the restriction of $\pi$ to $\left\{\exp t X_{j} \mid t \in \mathbf{R}\right\}$. Then for any $s>0$,

$$
\mathscr{H}(\pi)=\bigcap_{j=1}^{n} \mathcal{H}^{s}\left(\pi_{j}\right)
$$

In particular, if $0<s<1$, then $u \in \mathcal{H}^{s}(\pi)$ if and only if

$$
\|u\|+\sum_{j=1}^{n}\left\{\int_{-\infty}^{\infty}\left\|\pi\left(\exp t X_{j}\right) u-u\right\|^{2}|t|^{-2 s-1} d t\right\}^{1 / 2}<\infty,
$$

and (2.4) defines an equivalent norm on $\mathcal{H}^{3}(\pi)$.

REMARK. For integral $s,(2.3)$ was proved in [4, Theorem 5.2] for arbitrary $G$ and any basis $\left\{X_{j}\right\}$ for $g$.

EXAMPLE. Let $G$ be the " $a x+b$ " group of affine transformations of $\mathbf{R}$. $g$ has a basis $X_{1}, X_{2}$, with $\left[X_{1}, X_{2}\right]=X_{2}$. Let $\pi$ be the irreducible representation of $G$ on $\mathscr{H}=L^{2}(\mathbf{R} ; d x)$ such that $X_{1} \rightarrow d / d x$ and $X_{2} \rightarrow$ Multiplication by $i e^{x}$. Then for $s>0$,

$$
\begin{aligned}
& \mathcal{H}^{s}\left(\pi_{1}\right)=\text { usual } L^{2} \text { Sobolev space, } \\
& \mathcal{H}^{s}\left(\pi_{2}\right)=\left\{f \in L^{2} \mid e^{s x} f(x) \in L^{2}\right\},
\end{aligned}
$$

and $\mathcal{H}^{s}(\pi)=\mathcal{H}^{s}\left(\pi_{1}\right) \cap \mathcal{H}^{s}\left(\pi_{2}\right)$, with equivalent norm

$$
\|f\|_{s}^{2}=\int_{-\infty}^{\infty} e^{2 s x}|f(x)|^{2} d x+\int_{-\infty}^{\infty}(1+|\xi|)^{2 s}|\hat{f}(\xi)|^{2} d \xi
$$

$(\hat{f}=$ Fourier transform of $f)$. 
Corollary 2.3 (HyPOTHESES OF THEOREM 2.2). Let $m$ be a nonnegative integer and $0<\alpha<1$. Suppose $u \in \mathcal{H}(\pi)$ is such that for all $v \in \mathcal{H}(\pi)$ and $1<j<n$, the functions $\phi(t)=\left(\pi\left(\exp t X_{j}\right) u, v\right)$ are of class $C^{m}$ on $\mathbf{R}$, with $\phi^{(m)}$ Lipschitz continuous of exponent $\alpha$. Then $u \in \mathcal{F}^{m+s}(\pi)$ for all $s<\alpha$.

Proof. By Corollary 5.1 of [4] we have $u \in \mathcal{H}^{m}$. Set $w=\pi_{-\infty}\left(X_{j}^{m}\right) u$, where $\pi_{-\infty}$ denotes the representation of $U(\mathrm{~g})$ on the space $\mathcal{H}^{-\infty}(\pi)$ of continuous, conjugatelinear functionals on $\mathcal{H}^{\infty}(\pi)$. Then $w \in \mathcal{H}^{0}$, and for any $v \in \mathcal{H}$, one has $\left(\pi\left(\exp t X_{j}\right) w, v\right)=\phi^{(m)}(t)$, where $\phi(t)=\left(\pi\left(\exp t X_{j}\right) u, v\right)$. The Lipschitz continuity of $\phi^{(m)}$ and the uniform boundedness principle imply that

$$
\left\|\pi\left(\exp t X_{j}\right) w-w\right\|<C|t|^{\alpha}
$$

for some constant $C$ and $t \neq 0$. By (2.1) we conclude that $w \in \mathcal{F}^{s}\left(\pi_{j}\right)$, so that $u \in \mathcal{F}^{m+s}\left(\pi_{j}\right)$ for $1 \leqslant j \leqslant n$. Apply Theorem 2.2 .

Corollary 2.4 (Hypotheses of Theorem 2.2). Suppose $T \in U_{2 m}(\mathrm{~g})$ is elliptic, $u \in \mathcal{H}(\pi)$, and $\pi_{-\infty}(T) u \in \mathcal{H}^{k}(\pi)$ for some nonnegative integer $k$. Then $u \in$ $\mathcal{H}^{k+2 m-\varepsilon}(\pi)$ for all $\varepsilon>0$.

Proof. Let $v \in \mathcal{H}$. Set $w=\pi_{-\infty}(T) u, \phi(g)=(\pi(g) u, v)$, and $\psi(g)=$ $(\pi(g) w, v)$. Then $\psi \in C^{k}(G)$, and $\phi$ is a weak solution to the equation $T \phi=\psi$ on $G$. By classical elliptic regularity theory [1], $\phi \in C^{k+2 m-1}$, with all derivatives of order $k+2 m-1$ of $\phi$ being Lipschitz continuous of exponent $\alpha$, for any $\alpha<1$. Apply Corollary 2.3.

REMARK. Under the hypothesis that $T$ be associated with a Hermitian elliptic form in $U_{m}(\mathrm{~g}) \otimes U_{m}(\mathrm{~g})$, Corollary 2.4 holds with $\varepsilon=0$ and any real number $k$ (cf. [4], [5]).

3. Laplacians on Lie groups. Let $\left\{X_{j}\right\}$ be a basis for $\mathrm{g}$, and set $\Delta=\Sigma X_{j}^{2}$, acting as a left-invariant differential operator on $G$. Call $\Delta$ a Laplacian on $G$. For $t>0$, let $p_{t}(x)$ be the fundamental solution for the heat equation $u_{t}=\Delta u$ on $G$. Recall that a nonnegative Borel function $\phi$ on $G$ is submultiplicative if $\phi(x y)<\phi(x) \phi(y)$. For example, $\phi(x)=\|\pi(x)\|$ is submultiplicative, for any Banach space representation $\pi$ of $G$.

LEMMA 3.1. Let $\phi$ be submultiplicative on $G$. There are constants $M, \omega>0$ so that

$$
\int_{G} p_{t}(x) \phi(x) d x<M e^{\omega t}
$$

for all $t>0$.

Proof. See [8, §4].

REMARK. The notion of "Bessel potential", familiar in the case $G=\mathbf{R}^{n}$, can be defined in general using a Laplacian $\Delta$. Indeed, the operator $(\lambda-\Delta)^{-s}$ is bounded on $L^{2}(G ; d x)$ for $\operatorname{Re} s>0, \operatorname{Re} \lambda>0$, and acts by right convolution with the function

$$
J_{\lambda}^{s}(x)=\Gamma(s)^{-1} \int_{0}^{\infty} p_{t}(x) e^{-\lambda t} t^{s-1} d t
$$


From Lemma 3.1 one has

$$
\int_{G} \phi(x)\left|J_{\lambda}^{s}(x)\right| d x<M_{s}(\operatorname{Re} \lambda-\omega)^{-\operatorname{Re}(s)}
$$

if $\operatorname{Re} \lambda>\omega$. Thus for any Banach space representation $\pi$ of $G$, the resolvent of the semigroup $\pi\left(p_{t}\right)$ is $\pi\left(J_{\lambda}^{1}\right)$. Furthermore, when $\pi$ is unitary, then for any $\lambda>0$, $s>0$, one has

$$
\mathcal{H}^{s}(\pi)=\text { Range } \pi\left(J_{\lambda}^{s}\right)
$$

(cf. [4]).

4. Noncommutative interpolation. We recall some results about interpolation spaces associated with semigroups of operators ([2], [6]). Let $E$ be a Banach space, with norm $\|\cdot\|_{E}$. For $1 \leqslant p \leqslant \infty$, let $L_{*}^{p}(E)$ be the space of all strongly measurable functions $t \rightarrow u(t)$ from $(0, \infty)$ to $E$ such that

$$
\|u\|_{p, E}^{p}=\int_{0}^{\infty}\|u(t)\|_{E}^{p} \frac{d t}{t}<\infty
$$

$\left(\right.$ For $p=\infty,\|u\|_{\infty}=$ ess $\sup \|u(t)\|_{E}$.)

Suppose that $F \subset E$ is another Banach space, continuously embedded in $E$. If $0<\theta<1$, define $(F ; E)_{\theta, p}$ to be the subspace of all $x \in E$ which can be written as $x=u_{0}(t)+u_{1}(t)$, with $t^{-\theta} u_{0} \in L_{*}^{P}(F)$ and $t^{1-\theta} u_{1} \in L_{*}^{p}(E)$. Set

$$
\|x\|_{\theta, p}=\inf \left\{\left\|t^{-\theta} u_{0}\right\|_{p, F},\left\|t^{1-\theta} u_{1}\right\|_{p, E}\right\}
$$

the inf being taken over all such pairs $u_{0}, u_{1}$.

Example. Assume $F=\mathscr{D}(A)$, where $A$ is a closed operator on $E$ and $-A$ generates a strongly continuous semigroup $e^{-t A}$. By adding a large positive constant to $A$, we may assume that $(A+t)^{-1}$ exists as a bounded operator on $E$ for $t>0$. Then $x \in(\mathscr{D}(A) ; E)_{\theta, p}$ if and only if $t^{1-\theta} A(A+t)^{-1} x \in L_{*}^{p}(E)$, and the norm $\|x\|_{\theta, p}$ is equivalent to

$$
\|x\|_{E}+\left\{\int_{0}^{\infty}\left\|t^{1-\theta} A(A+t)^{-1} x\right\|_{E}^{p} \frac{d t}{t}\right\}^{1 / p} .
$$

In particular, if $E$ is a Hilbert space and $A \geqslant I$ is selfadjoint, then by (4.1) one sees that

$$
(\mathscr{D}(A) ; E)_{\theta, 2}=\mathscr{D}\left(A^{1-\theta}\right)
$$

for $0<\theta<1$, with equivalent norm $\left\|A^{1-\theta} u\right\|_{E}$.

Suppose now that $A$ and $B$ are closed operators on $E$, with $-A$ and $-B$ each generating strongly continuous semigroups. Assume that there are constants $C, \omega$ $>0$ with

$$
\begin{gathered}
e^{-t A}: \mathscr{D}(B) \rightarrow \mathscr{D}(B) \text { for } t>0 . \\
\left\|B e^{-t A} x\right\|_{E}<C e^{\omega t}\left\{\|x\|_{E}+\|B x\|_{E}\right\}
\end{gathered}
$$

for $x \in \mathscr{D}(B)$ and $t>0$. 
Theorem 4.1 (Grisvard [7]). Set $F=\mathscr{D}(A) \cap \mathscr{D}(B)$. Then for $0<\theta<1$ and $1 \leqslant p \leqslant \infty$, one has

$$
(F ; E)_{\theta, p}=(\mathscr{D}(A) ; E)_{\theta, p} \cap(\mathscr{D}(B) ; E)_{\theta, p} .
$$

Remark. In [7] this result is stated with $C e^{\omega t}$ replaced by $C$ in (4.4). This can be achieved by replacing $A$ by $A+\omega I$, of course, without changing $\mathscr{D}(A)$.

5. Proof of the Reduction Theorem. Take Laplacians $\Delta_{H}$ and $\Delta_{K}$ on $H$ and $K$, respectively, and denote by $p_{t}^{H}$ and $p_{t}^{K}$ the corresponding fundamental solutions to the heat equations on $H$ and $K$. Let $\sigma=\left.\pi\right|_{H}, \tau=\left.\pi\right|_{K}$, and take $A=$ closure of $\sigma_{\infty}\left(-\Delta_{H}\right), B=$ closure of $\tau_{\infty}\left(-\Delta_{K}\right)$, where for any representation $\pi$ of $G, \pi_{\infty}$ denotes the representation of $U(\mathfrak{g})$ on $\mathcal{H}^{\infty}(\pi)$. Then $-A$ and $-B$ are the generators of the semigroups $\sigma\left(p_{t}^{H}\right)$ and $\tau\left(p_{t}{ }^{K}\right)$, respectively. Furthermore, for $s>0$,

$$
\mathscr{H}(\sigma)=\mathscr{D}\left(A^{s / 2}\right), \quad \mathscr{C}(\tau)=\mathscr{D}\left(B^{s / 2}\right)
$$

and by [4, Theorem 5.2],

$$
\mathcal{H}^{2}(\pi)=\mathscr{D}(A) \cap \mathscr{D}(B) .
$$

Set $E=\mathcal{H}(\pi), F=\mathcal{H}^{2}(\pi)$. To verify (4.3) in this situation, it suffices by (5.1) to show that $\sigma\left(p_{t}^{H}\right)$ leaves $\mathcal{H}^{2}(\tau)$ invariant. But since $K$ is a normal subgroup of $G$, $\mathcal{H}^{2}(\tau)$ is clearly invariant under $\pi(g), g \in G$, and $G$ acts continuously on $\mathcal{H}^{2}(\tau)$, with $\|\tau(g) v\|_{\mathcal{K}^{2}(\tau)} \leqslant \phi(g)\|v\|_{\mathcal{K}^{2}(\tau)}$, where $\phi(g)=\left\|\left.\operatorname{Ad}\left(g^{-1}\right)\right|_{U_{2}(\mathfrak{l})}\right\|$. By Lemma 3.1 it is clear that the integral

$$
\int_{H} p_{t}^{H}(h) \pi(h) v d h=\sigma\left(p_{t}^{H}\right) v
$$

converges absolutely in the norm of $\mathcal{H}^{2}(\tau)$, and

$$
\left\|\sigma\left(p_{t}^{H}\right) v\right\|_{\mathcal{F}^{2}(\tau)} \leqslant C e^{\omega t}\|v\|_{\mathcal{X}^{2}(\tau)}
$$

for $v \in \mathcal{H}^{2}(\tau)$. By (5.1) this also establishes (4.4). Taking $p=2$ in Theorem 4.1, we conclude by (5.1) and (4.2) that

$$
\mathcal{H}^{S}(\pi)=\mathcal{H}^{\Im}(\sigma) \cap \mathcal{H}^{\Im}(\tau)
$$

for $0<s<2$.

To establish (5.2) in general, we observe that by Theorem 5.2 of [4], $u \in$ $\mathcal{H}^{2 m+s}(\pi)$ if and only if $\pi_{-\infty}(R) u \in \mathcal{H}^{2}(\pi)$, where $R=\Sigma X_{j}^{2 m}$ (m a positive integer, $\left\{X_{j}\right\}$ a basis for $\left.\mathfrak{g}\right)$. Choosing $\left\{X_{j}\right\}$ as the union of bases for $\mathfrak{h}$ and $\mathfrak{f}$, we see that $u \in \mathscr{H}^{2 m+s}(\pi)$ if and only if $\pi_{-\infty}(S) u \in \mathcal{H}^{s}(\pi)$ and $\pi_{-\infty}(T) u \in \mathcal{H}^{s}(\pi)$ for all $S \in U_{2 m}(\mathfrak{h})$ and $T \in U_{2 m}(\mathfrak{f})$. Hence if $0<s<2$ and $u \in \mathcal{H}^{2 m+s}(\sigma) \cap \mathcal{H}^{2 m+s}(\tau)$, it follows by (5.2) that $u \in \mathcal{H}^{2 m+s}(\pi)$. The opposite inclusion is evident by the monotonicity property of interpolation, which completes the proof.

\section{REFERENCES}

1. L. Bers, F. John and M. Schechter, Partial differential equations, Interscience, New York, 1964.

2. P. L. Butzer and H. Berens, Semigroups of operators and approximation, Springer-Verlag, New York and Berlin, 1967.

3. R. Goodman, One-parameter groups generated by operators in an enveloping algebra, J. Functional Analysis 6 (1970), 218-236. 
4. Some regularity theorems for operators in an enveloping algebra, J. Differential Equations 10 (1971), 448-470.

5. (1980), 763-777.

6. P. Grisvard, Commutativité de deux foncteurs d'interpolation et applications, J. Math. Pures Appl. 45 (1966), 143-290.

7. , Interpolation non-commutative, Atti Accad. Naz. Lincei Rend. C. Sci. Fis. Mat. Natur. 52 (1972), 11-15.

8. A. Hulanicki, Subalgebra of $L_{1}(G)$ associated with Laplacian on a Lie group, Colloq. Math. 31 (1974), 259-287.

9. R. S. Palais et al., Seminar on the Attiyah-Singer index theorem, Ann. of Math. Studies, no. 57, Princeton Univ. Press, Princeton, N.J., 1965.

10. E. M. Stein, Singular integrals and differentiability properties of functions, Princeton Univ. Press, Princeton, N.J., 1970.

Department of Mathematics, Rutgers University, New Brunswick, New Jersey 08903 